\title{
FARMACOLOGIA DE LA SOLIFENACINA
}

\author{
Francisco J. Morales-Olivas y Luis Estañ.
}

Departamento de Farmacología. Facultad de Medicina y Odontología. Universidad de Valencia. Valencia. España.

\begin{abstract}
Resumen.- Los antimuscarínicos son los fármacos de primera elección para el tratamiento del síndrome de vejiga hiperactiva y su relación beneficio/riesgo depende en gran medida de la selectividad por los distintos subtipos de receptores muscarínicos. Solifenacina es el antimuscarínico que muestra mayor selectividad por los receptores $M 3$ vesicales, lo que puede traducirse en una menor incidencia de reacciones adversas relacionadas con otros subtipos de receptor. Las vías metabólicas de los antimuscarínicos pueden influir en la eficacia y en la aparición de interacciones. Solifenacina es metabolizada sólo por el CYP3A4 originando tres metabolitos inactivos y uno con actividad similar a la del compuesto original. Sin embargo, otros fármacos del grupo son también sustrato para el CYP 2D6 que presenta polimorfismos, por lo que su cinética se puede modificar en los pacientes metabolizadores lentos. El riesgo de
\end{abstract}

\section{CORRESPONDENCIA}

Francisco J. Morales-Olivas

Departamento de Farmacología Facultad de Medicina y Odontología Universidad de Valencia Avda. Blasco Ibáñez, 15 46010 Valencia (España).

morales@uv.es

Aceptado para publicar: 20 de diciembre 2009. interacciones de solifenacina es bajo, e inferior al de los antimuscarínicos que se metabolizan también por el CYP 2D6. La fracción inalterada de solifenacina que se elimina por orina junto con el metabolito activo pueden contribuir al efecto terapéutico actuando sobre los receptores del urotelio. No es necesario ajustar las dosis de solifenacina en pacientes de edad avanzada o con insuficiencia hepática o renal moderadas.

Palabras clave: Antimuscarínicos. Vejiga hiperactiva. Solifenacina. Farmacocinética.

Summary.- Antimuscarinics are the drugs of choice for the treatment of overactive bladder syndrome, and their benefit/risk ratio depends largely on selectivity for the different subtypes of muscarinic receptors. Solifenacin is the antimuscarinic that presents greatest selectivity for M3 bladder receptors, which may translate into a lower incidence of undesirable effects related to other receptor subtypes. Metabolic pathways of the antimuscarinics may impact efficacy and appearance of interactions. Solifenacin is metabolized only by the CYP3A4, giving three inactive metabolites and one with a similar activity to the original compound. However, other drugs in the group are also a substrate for the CYP 2D6 which presents polymorphisms, whereby their pharmacokinetics may be modified in slow metabolizers. The risk of interactions of solifenacin is low, and it is lower than that of the antimuscarinics which are also metabolized by the CYP 2D6. The unaltered fraction of solifenacin which is eliminated in urine, together with the active metabolite, can contribute to the therapeutic effect by acting on the urothelium receptors. It is not necessary to adjust doses of solifenacin in elderly patients or those with moderate liver or kidney impairment.

Keywords: Antimuscarinics. Overactive bladder. Solifenacin. Pharmacokinetics. 


\section{INTRODUCCIÓN}

El Síndrome de Vejiga Hiperactiva (SVH) se definió por la International Continence Society (ICS) como: urgencia miccional, con o sin incontinencia de urgencia, generalmente acompañada de frecuencia y nocturia en ausencia de infección probada $u$ otra enfermedad demostrable (1). Estas combinaciones de síntomas son sugestivas de hiperactividad del detrusor demostrable urodinámicamente, pero pueden ser debidas a otras formas de disfunción uretro-vesical. Estos términos pueden ser usados si no existe infección probada u otra patología.

Puede afectar a ambos sexos, con predominio en las mujeres, aumenta su incidencia con la edad y altera la calidad de vida de quien la padece. La prevalencia en Europa oscila entre el $12 \%$ y el $22 \%$ en personas de 40 o más años de edad (2). En el estudio NOBLE, la prevalencia encontrada fue del 16,5\% de la población mayor de 18 años (3). Un estudio epidemiológico realizado en España, registró una prevalencia de sintomatología compatible con $\mathrm{SVH}$, en personas de edad igual o superior a los 40 años, del $21,5 \%$ (4).

El síntoma principal de la vejiga hiperactiva es la urgencia miccional, causada por las contracciones involuntarias del músculo detrusor, que se producen cuando la vejiga se está llenando.

EL SVH altera la calidad de vida y suele asociarse a problemas sociales, psicológicos, laborales, físicos y sexuales. Las personas que la padecen están expuestas a un riesgo mayor de irritación, ulceración e infección en la piel y mucosas del tracto genitourinario.

Para mejorar los síntomas, los pacientes deben recibir educación acerca de la función vesical para modificar su conducta e instaurar una micción programada o profiláctica. Mediante la reeducación vesical y la realización de ejercicios del suelo de la pelvis, se puede aumentar progresivamente los intervalos entre micciones.

Desde el punto de vista farmacológico, el objetivo será inhibir las contracciones vesicales del músculo detrusor, disminuir la sensibilidad vesical y la frecuencia miccional, con el consecuente incremento en la capacidad de la vejiga para almacenar orina. No existen fármacos, por el momento, capaces de corregir todos los aspectos relacionados con esta enfermedad multifactorial. Sin embargo, los antimuscarínicos, por su capacidad para bloquear la actividad de los receptores colinérgicos ubicados en la vejiga, son la opción principal para el tratamiento del SVH.
Estos fármacos son ampliamente utilizados y su eficacia frente a placebo ha sido demostrada en múltiples ensayos clínicos aleatorizados.

Sin embargo, los antimuscarínicos también se relacionan con la aparición de efectos adversos que inciden en el cumplimiento y la persistencia del tratamiento a largo plazo. Con los nuevos productos, se busca que la relación entre efectos beneficiosos y efectos negativos se incline a favor de los primeros.

\section{MECANISMO DE ACCIÓN}

El sistema nervioso autónomo controla las funciones viscerales del organismo y su misión es mantener la homeostasia en respuesta tanto a las alteraciones del medio interno como a los estímulos exteriores.

El sistema nervioso autónomo se divide en dos partes: el simpático o adrenérgico y el parasimpático o colinérgico. La contracción de la vejiga se controla principalmente por el parasimpático cuyo neurotransmisor es la acetilcolina. Este neurotransmisor actúa sobre los receptores muscarínicos distribuidos por el músculo detrusor, originando la contracción del mismo lo que aumenta la presión en la vejiga y causa, a su vez, la micción.

Se han identificado hasta 5 subtipos de receptores muscarínicos denominados $M 1, M 2, M 3$, M4 y M5 tanto en el sistema nervioso central (SNC) como en los tejidos periféricos. Los receptores muscarínicos pertenecen a la familia de receptores acoplados a proteínas $G$. Están ampliamente distribuidos en el organismo y la mayoría de los tejidos tienen más de un subtipo (Tabla I). La identificación de receptores se ha realizado mediante técnicas de biología molecular y marcado radioactivo. Sólo para algunos subtipos existen además pruebas funcionales. Ello explica que a pesar de conocer su ubicación, se desconozca la función específica en algunos territorios.

A nivel del tracto urinario predominan los receptores $M 2$ y $M 3$, en una relación de 3:1. La contracción del músculo detrusor se debe fundamentalmente a los efectos de la acetilcolina sobre los receptores M3 ya que aunque los receptores $M 2$ son predominantes en la vejiga urinaria y anejos, los receptores M3 son los más importantes desde el punto de vista funcional y su estímulo es el responsable directo de la contracción del músculo detrusor (6).

Aunque ambos subtipos están acoplados a proteínas $G$, sus vías de transducción de señales son diferentes (7). La estimulación de los receptores M3 
TABLA I. SUBTIPOS DE RECEPTORES MUSCARÍNICOS Y LOCALIZACIÓN DE LOS MISMOS EN EL ORGANISMO (5).

\begin{tabular}{|c|c|}
\hline RECEPTOR & LOCALIZACIÓN \\
\hline M1 & Glándulas salivares, Corazón, Cerebro, Ojos \\
\hline M2 & Vejiga, Tracto gastrointestinal, Corazón, Cerebro, Ojos \\
\hline$M 3$ & Vejiga, Tracto gastrointestinal, Glándulas salivares, Corazón, Cerebro, Ojos \\
\hline$M 4$ & Cerebro, Ojos \\
\hline$M 5$ & Corazón, Cerebro, Ojos \\
\hline
\end{tabular}

produce la contracción del detrusor a través de hidrólisis de los fosfoinositoles (IP3), aunque también se ha implicado a canales de cationes no selectivos, a la participación del ion calcio y a la activación de la Rho-quinasa. Los resultados de distintos estudios experimentales sugieren que la contracción mediada por receptores M3 en el músculo detrusor humano es dependiente de la entrada de calcio a través de los canales lentos de calcio y a la inhibición de la cadena ligera de la miosina a través de la activación de Rho-quinasa y proteín-quinasa $C$ pudiendo también participar canales de potasio $(8,9)$.

Aunque la contribución de los receptores M2 a la contracción del detrusor es menor que la de receptores $M 3$ en la vejiga sana, en animales de experimentación la contribución de los $M 2$ podría ser mayor en algunos cuadros patológicos, pero estos datos no han sido confirmados en humanos (10) (Figura 1).

La vejiga urinaria también tiene inervación simpática y los receptores beta3-adrenérgicos están implicados en la dinámica miccional, su estímulo da lugar a relajación del detrusor. Estos receptores están localizados además de en la vejiga, en la próstata, el colon, el músculo esquelético, el corazón y el tejido adiposo y participan en funciones metabólicas de las catecolaminas endógenas, como lipólisis, termogénesis, procesos de motilidad del tracto gastrointestinal y urinario (1 1). El desarrollo clínico de agonistas $\beta 3$ adrenérgicos para el tratamiento de la hiperactividad vesical es una alternativa de futuro que puede contribuir a mejorar este cuadro patológico completando el arsenal terapéutico disponible hasta el momento.

Todos los subtipos de receptores muscarínicos han sido detectados en el urotelio y en la capa suburotelial de humanos $(12,13)$. El receptor $M 2$ se localiza en las células en paraguas (umbrella cells), el M1 en la capa basal, mientras que el M3 está distribuido más homogéneamente. Se ha sugerido que el estímulo de los receptores muscarínicos uroteliales pudiera estar involucrado en la generación de impulsos aferentes (14).

Se ha observado un aumento significativo de la inmunorreactividad a receptores $M 2$ y $M 3$ en urotelio y en miofibroblastos del suburotelio de pacientes con hiperactividad idiopática del músculo detrusor en comparación con individuos control. Esta inmunorreactividad se correlaciona de forma significativa con la sensación de urgencia y con la frecuencia miccional en estos pacientes, lo que sugiere un papel potencial de estos receptores en los mecanismos fisiopatológicos y en el efecto terapéuti-

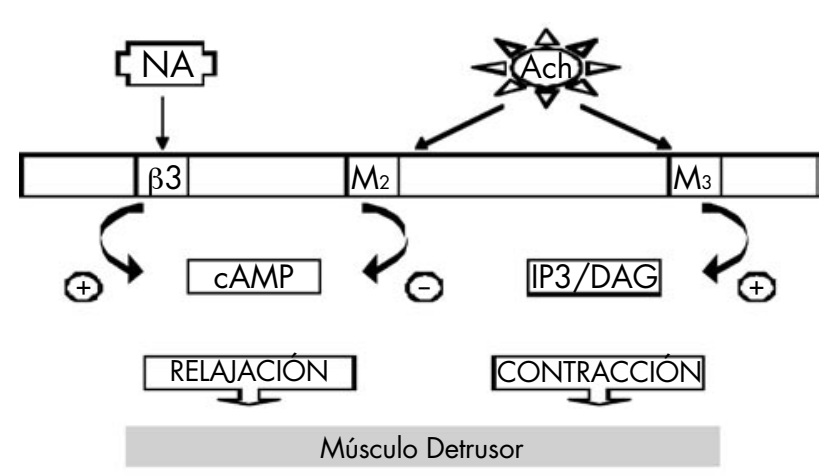

FIGURA 1. Mecanismos y vías principales de activación de los receptores muscarínicos del músculo detrusor. (Adaptado de 6)

$N A=$ Noradrenalina. Ach $=$ Acetilcolina. $B 3=$ Receptor beta3. M2= Receptor muscarínico M2. M3= Receptor muscarínico M3. $c A M P=A M P$ cíclico. IP3=Inositol trifosfato. DAG= Diacilglicerol. += Activación. -=Inhibición. 
co de los antimuscarínicos (15). La activación de los receptores muscarínicos de las células uroteliales da lugar a liberación de ATP que activa fibras nerviosas aferentes capaces de alterar la excitabilidad nerviosa a este nivel comportándose como un transmisor neuromuscular excitador $(16,17)$. Además, en cultivos de células uroteliales, el bloqueo de los receptores muscarínicos con atropina inhibe la liberación de ATP mediada por la distensión que desencadena el reflejo de micción (18).

El sistema colinérgico y los receptores muscarínicos están ampliamente distribuidos por el sistema urinario, desde el músculo detrusor a los miocitos y las estructuras internas de la mucosa como los nervios aferentes (sensoriales). Los receptores uroteliales y suburoteliales están involucrados en señales aferentes de la vejiga, pero no se ha establecido totalmente en qué medida esta vía de señalización participa en el reflejo miccional, y por tanto qué efectos se podrían esperar de su bloqueo. Algunos antimuscaríni$\cos$ y sus metabolitos activos se excretan en la orina en cantidades que pueden bloquear los receptores muscarínicos de la mucosa y aunque aún no se ha demostrado que su bloqueo incremente la eficacia clínica, estos hallazgos sugieren que actuar sobre las vías aferentes del urotelio puede ser interesante desde el punto de vista terapéutico (19).

El tratamiento de los pacientes con $\mathrm{SVH}$ va dirigido fundamentalmente al alivio de los síntomas (urgencia, incontinencia de urgencia, polaquiuria y nocturia), siendo los fármacos antimuscarínicos el eje del tratamiento farmacológico. Estos fármacos son antagonistas competitivos de los receptores muscarínicos y actúan bloqueando el estimulo colinérgico por lo que reducen las contracciones del detrusor. El efecto terapéutico se produce fundamentalmente durante la fase de llenado del ciclo miccional. Disminuyen la sensación de urgencia miccional, retrasan la micción y reducen, las contracciones del detrusor. Durante la fase de vaciado, su papel como depre-

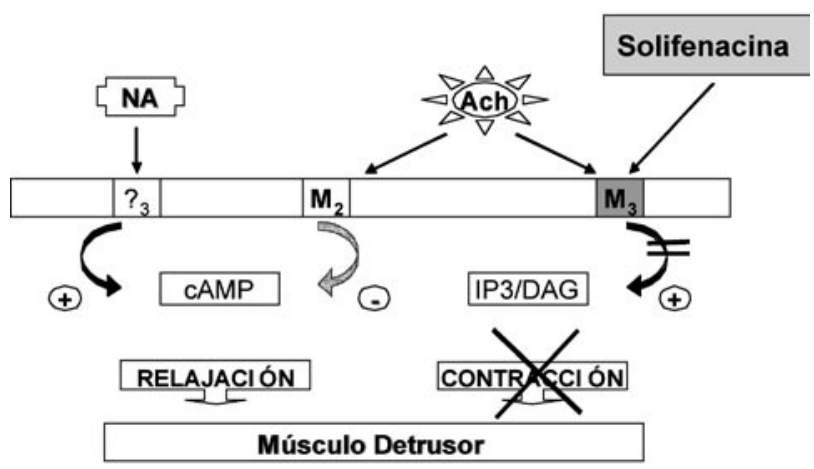

FIGURA 2. Mecanismo de acción de solifenacina (Adaptado de 6). sores de la capacidad contráctil del detrusor parece más limitado $(20,21)$.

Solifenacina es un antagonista competitivo de los receptores muscarínicos con elevada especificidad y selectividad sobre los receptores $M 3$, que son los funcionalmente importantes en la actividad vesical (Figura 2).

Hablamos de especificidad de un fármaco para referirnos a su afinidad o capacidad para unirse a un tipo de receptor. La afinidad se mide por medio de la dosis (concentración) necesaria para activar o bloquear el receptor, cuanto menor sea la dosis que produce el efecto mayor es la afinidad. Y cuanto mayor sea la afinidad mayor es la especificidad.

Cuando existen distintos tipos de receptores, como es el caso de los muscarínicos, la selectividad se mide por el cociente entre las concentraciones necesarias para estimular o bloquear a dos de ellos. Lo importante es el cociente, no la concentración.

Diferentes estudios, tanto in vitro como in vivo, indican que solifenacina es un inhibidor competitivo muy específico de los receptores muscarínicos M3 (Tabla II), porque los inhibe con concentraciones muy bajas. En estudios realizados en humanos (2224) para calcular la dosis que inhibe los distintos subtipos de receptores se puso de manifiesto que la solifenacina bloqueaba los receptores M3 a concentración de $12 \mathrm{nM}$ mientras que para bloquear los otros subtipos de receptores necesitaba concentraciones más altas. El cociente entre concentraciones es el siguiente $M 1 / M 3=2,2 ; M 2 / M 3=14,2 ; M 4 /$ $M 3=9,2 ; M 5 / M 3=2,6$. Ello significa que son necesarias concentraciones entre 2,2 y 14,2 veces superiores a las que bloquean el M3 para bloquear a los otros subtipos de receptores muscarínicos.

Los agentes con mayor selectividad por la vejiga pueden tener mejor tolerabilidad. Solifenacina es el antimuscarínico con mayor especificidad por los receptores $M 3(26,27)$.

En glándulas salivares de ratas y monos, solifenacina produce menor efecto bloqueante que otros antimuscarínicos y la concentración necesaria para reducir la secreción salival es entre 3,6 y 6,5 veces superior a la que produce efectos en vejiga $(28,29)$. Por ello, se ha señalado que la gran selectividad de solifenacina por la vejiga en comparación con otros antimuscarínicos no puede explicarse únicamente por su mayor afinidad por los receptores $M 3$ que por los receptores M1 y M5 y deben existir otros mecanismos que todavía no están investigados adecuadamente en humanos $(27,30)$. 
TABLA II. AFINIDAD POR LOS RECEPTORES MUSCARÍNICOS. VALORES Ki (nM) EN RECEPTORES MUSCARÍNICOS RECOMBINANTES HUMANOS.

\begin{tabular}{|l|c|c|c|c|c|}
\hline & M1 & M2 & M3 & M4 & M5 \\
\hline FESOTERODINA (25) & 631 & 501 & $>1000$ & 158 & $>1000$ \\
\hline 5-HMT (metabolito activo de fesoterodina) (25) & 2.3 & 2.0 & 2.5 & 2.8 & 2.9 \\
\hline OXIBUTININA (24) & 6.1 & 21 & 3.4 & 6.6 & 18 \\
\hline SOLIFENACINA (24) & 26 & 170 & 12 & 110 & 31 \\
\hline TOLTERODINA (24) & 2.7 & 4.2 & 4.4 & 6.6 & 2.5 \\
\hline
\end{tabular}

Ki $(n M)=$ concentración inhibitoria 50\% expresada en nanomolar. Concentración del antimuscarínico que reduce al 50\% el efecto de acetilcolina sobre el receptor correspondiente.

Se realizó un estudio (31) con el objetivo de evaluar los efectos locales de tres antimuscarínicos, para ello se analizó el efecto urodinámico de la orina humana administrada intravesicalmente a ratas hembras a las que se inducía hiperactividad vesical con carbacol. Carbacol redujo significativamente el intervalo entre contracciones y la capacidad de la vejiga en el grupo control, que recibía orina en ausencia de antimuscarínicos, y en los de tolterodina o darifenacina. Sin embargo, la administración de orina humana después de tomar solifenacina inhibió la hiperactividad del detrusor inducida por carbacol. Estos resultados permiten concluir que solifenacina posee efectos locales en el urotelio que suponen una ventaja para el tratamiento del $\mathrm{SVH}$.

\section{FARMACOCINÉTICA DE LA SOLIFENACINA}

La farmacocinética es la parte de la farmacología que estudia el recorrido de un fármaco a través del organismo de los seres vivos y las modificaciones que éstos producen sobre el fármaco. Los procesos farmacocinéticos se agrupan bajo el acrónimo LADME construido con la inicial de cada uno de ellos (Liberación, Absorción, Distribución, Metabolismo y Excreción). La absorción es el paso del fármaco del exterior al interior del organismo, cuando la administración es oral ello supone ir desde la luz intestinal al plasma. La distribución se refiere al modo en que el fármaco es transportado en el plasma y a como se reparte en los distintos órganos y tejidos. Mediante el metabolismo el fármaco es transformado para facilitar su excreción y en ocasiones su acción. La excreción es el proceso inverso a la absorción. Metabolismo y excreción, en conjunto, constituyen el proceso de eliminación. Aunque habitualmente se presta más atención a los pasos comprendidos entre la absorción y la excreción, la liberación del fármaco desde la forma farmacéutica en que se administra es el primer proceso, en el caso de la administración oral se produce en la luz del tubo digestivo, y es imprescindible para que el fármaco pueda absorberse. Los distintos procesos cinéticos suponen que el fármaco atraviese membranas celulares, por ello la farmacocinética de una sustancia viene determinada por sus propiedades fisico-químicas, como peso molecular y liposolubilidad, en tanto que ésta depende del grado de ionización, tiene también influencia el pH del medio.

Los estudios de farmacocinética de un medicamento se realizan generalmente en voluntarios sanos y forman parte de la fase I de los ensayos clínicos, a veces continúan durante la fase II en pacientes con la enfermedad a la que el fármaco va dirigido. Se suelen hacer también estudios en situaciones en que los parámetros farmacocinéticos pueden modificarse como en la insuficiencia renal y hepática y en pacientes de edad avanzada.

La farmacocinética de la solifenacina ha sido estudiada en adultos sanos, jóvenes y de edad avanzada, y en pacientes con insuficiencia hepática y renal. Se ha estudiado tanto la administración de dosis únicas como de dosis múltiples (32-34).

\section{Liberación}

La solifenacina se presenta en comprimidos de 5 y $10 \mathrm{mg}$ de liberación inmediata, lo que significa que el principio activo se libera desde la forma farmacéutica a partir del momento en que entra en 
contacto con el líquido presente en el tubo digestivo. Otros antimuscarínicos como fesoterodina y tolterodina se administran en comprimidos de liberación prolongada que hace que el proceso se produzca más lentamente.

\section{Absorción y biodisponibilidad}

La solifenacina, como otros antimuscarínicos que se administran por vía oral, es una amina terciaria lo que hace que pueda atravesar con facilidad las membranas biológicas. Después de la administración de un comprimido de solifenacina se detecta fármaco en plasma de forma casi inmediata, consiguiéndose la concentración plasmática máxima (Cmax) entre 3 y 8 horas después de la administración con independencia de la dosis utilizada (35). El paso a plasma es rápido porque solifenacina, debido a su liposolubilidad y su pequeño tamaño molecular, pasa las membranas por difusión pasiva, no es afectada por enzimas del interior del enterocito, ni es sustrato para la P-glicoproteína. Estudios en animales muestran que no se absorbe en el estómago, haciéndolo en todas las porciones del intestino delgado (30). La absorción no se afecta por los alimentos y las concentraciones obtenidas son semejantes cuando se administra antes o después de las comidas (36).

Cuando se administran dosis repetidas cada 24 horas se consigue concentraciones plasmáticas estables (equilibrio estacionario) al cabo de 10 días (30).

La biodisponibilidad mide la cantidad de fármaco que está en condiciones de ejercer su acción, ello se hace mediante la determinación seriada de niveles plasmáticos para construir una gráfica de éstos respecto al tiempo. El área comprendida bajo la curva ( $A B C$ ○ $A U C$ en inglés) es el valor de la biodisponibilidad en magnitud, mientras que el tmax, tiempo que se tarda en alcanzar la Cmax, mide la biodisponibilidad en velocidad. La biodisponibilidad relativa compara los valores de una misma sustancia administrada por dos vías, de las que la intravenosa (i.v.) es siempre la que sirve como patrón. La biodisponibilidad de solifenacina ha sido estudiada con dosis de $10 \mathrm{mg}$ administradas por vía oral frente a dosis de $30 \mathrm{mg}$ i.v. Se recogieron muestras de niveles plasmáticos durante 192 horas y se calculó que administrada por vía oral tiene una biodispobilidad media del 88\% [IC95\% 75,8\%-102,1\%], existe una variación interindividual del 13\% (33). Todo ello permite afirmar que la biodisponibilidad de solifenacina es elevada. Además, es mayor y menos variable que la de otros antimuscarínicos como fesoterodina, tolterodina y oxibutinina $(37,38)$.

La biodisponibilidad cuando se administran dosis múltiples (situación habitual en la clínica) es se- mejante a la de dosis únicas. El perfil del $A B C$ de 24 horas cuando se ha conseguido equilibrio estacionario (concentraciones plasmáticas estables después de administración múltiple) es comparable al perfil de 24 horas conseguido con la primera dosis (30). Este dato demuestra que no existe acumulación de principio activo después de la administración repetida.

\section{Distribución}

La solifenacina se reparte de forma amplia en el organismo, desde el plasma pasa a otros órganos y sistemas. Circula en el plasma unida, en un 93-96\%, a proteínas plasmáticas, se une a la alfalglicoproteína pero no a la albúmina, lo que hace que el riesgo de interacciones con otros fármacos transportados por ésta sea nulo. La unión es reversible y la proporción entre fracción libre y fracción unida es independiente de la dosis y de las características del paciente (34).

El volumen aparente de distribución, que indica de la forma en que se distribuye, es elevado, $599 \pm 86$ litros después de la administración de $5 \mathrm{mg}$ $(33,39)$. Se puede afirmar que solifenacina se distribuye de forma amplia. Se detectan concentraciones elevadas en el riñón y el hígado, órganos encargados de la eliminación, y en la vejiga urinaria donde ejerce su acción terapéutica.

No hay datos del paso de solifenacina a líquido cefalorraquídeo en humanos, el estudio SCOPE (40) demostró que no afecta a los procesos cognitivos en sujetos sanos de edad avanzada. En ratas puede pasar la barrera hematoencefálica pero no afecta al aprendizaje. Estos resultados se pueden explicar por su mayor selectividad por los receptores $M 3$ que por los M1, implicados en procesos cognitivos (41).

\section{Metabolismo}

Solifenacina se metaboliza fundamentalmente en el hígado. Sufre procesos de oxidación, hidroxilación y glucuronoconjugación, siendo el más importante la oxidación. Se producen cuatro metabolitos, el más abundante es la 4-R-hidroxisolifenacina (M3) que se forma por acción de la isoforma $3 \mathrm{~A} 4$ del citocromo $\mathrm{P} 450$, responsable también de la formación del metabolito $M 4$, el metabolito $M 2$ es consecuencia de la participación de otras isoformas, mientras que el M5, que es el menos abundante, se forma por conjugación con ácido glucurónico. Los metabolitos M2, M4 y M5 son inactivos. El metabolito M3 tiene actividad farmacológica similar a la del compuesto original, aunque su concentración es baja, podría contribuir al efecto terapéutico $(34,35)$.

Otros antimuscarínicos como tolterodina (42) o fesoterodina (43) son metabolizados, también, por 
el CYP 2D6. Existen polimorfismos genéticos para CYP 2D6, lo que significa que hay grandes diferencias interindividuales en la velocidad de metabolización. Un 7\% de la población blanca metaboliza de forma lenta. En el caso de la tolterodina el CYP 2D6 convierte al fármaco en su metabolito activo, que tiene una gran participación en el efecto farmacológico. La ruta metabólica para los metabolizadores lentos es la desalquilación vía CYP3A4 que da lugar a tolterodina $\mathrm{N}$-desalquilada, que carece de efecto. El aclaramiento reducido produce concentraciones séricas de tolterodina significativamente más altas (unas 7 veces).

En el caso de fesoterodina que es un profármaco carente de actividad farmacológica, la conversión en metabolito activo se produce en el plasma por acción de las esterasas, pero posteriormente el CYP 2D6 da lugar a metabolitos inactivos. Como se ha comentado un $7 \%$ de la población metaboliza lentamente, lo que puede dar lugar a un incremento de los niveles plasmáticos del metabolito activo con el consiguiente riesgo de aparición de reacciones adversas dosis dependientes.

\section{Excreción}

La excreción de solifenacina se produce sobre todo por riñón. Después de la administración de una dosis única se recoge en orina aproximadamente un $15 \%$ de fármaco sin modificar, $17,8 \%$ del metabolito $M 2,8,3 \%$ del $M 3,8,9 \%$ del $M 4$ y $0,4 \%$ de M5. Después de la administración de dosis repetidas se recoge en orina de 24 horas entre el 8 y el $13 \%$ de solifenacina sin modificar (44).

La vida media de eliminación es prolongada y no dependiente de la dosis administrada. Se han descrito valores entre 36 y 66 horas en administración única y entre 33 y 85 horas tras administración repetida. La variabilidad del resultado cabe atribuirla a los distintos métodos empleados en los diferentes estudios. En cualquier caso se trata de un valor supe-

TABLA III. CARACTERÍSTICAS FARMACOCINÉTICAS DE TOLTERODINA, SOLIFENACINA Y FESOTERODINA.

\begin{tabular}{|c|c|c|c|}
\hline & Tolterodina & Solifenacina & Fesoterodina \\
\hline Presentación & $\begin{array}{c}\text { Capsulas liberación } \\
\text { prolongada }\end{array}$ & $\begin{array}{l}\text { Comprimidos } \\
\text { recubiertos }\end{array}$ & $\begin{array}{c}\text { Comprimidos liberación } \\
\text { prolongada }\end{array}$ \\
\hline Biodisponibilidad & $17-65 \%$ & $\approx 90 \%$ & $52 \%$ (5 HMT derivado) \\
\hline tmáx & 2-6 horas & 3-8 horas & 5 horas \\
\hline Distribución & Vd $113 \mathrm{~L}$ & $\begin{array}{l}\text { UPP } 98 \%(\alpha 1 \mathrm{gp}) \\
\qquad \mathrm{Vd} 600 \mathrm{~L}\end{array}$ & $\begin{array}{c}\text { UPP } 50 \% \text { ( } \alpha 1 \text { gp y albúmina) } \\
\text { Vd } 169 \mathrm{~L}\end{array}$ \\
\hline Metabolismo & $\begin{array}{c}\text { Primer paso CYP 2D6 } \\
\text { Hepático CYP3A4 y } \\
\text { CYP2D6* }\end{array}$ & Hepático CYP3A4 & Hepático CYP3A4 y CYP2D6* \\
\hline Excreción & $\begin{array}{c}\text { Orina }(\approx 77 \%) \text { y heces. } \\
<1 \% \text { inalterado }\end{array}$ & $\begin{array}{c}\text { Orina }(\approx 70 \%) \text { y heces } \\
11 \% \text { inalterado } \\
8 \% \text { metabolito activo }\end{array}$ & $\begin{array}{l}\text { Orina }(\approx 70 \%) \text { y heces } \\
16 \% \quad(5 \mathrm{HMT} \text { derivado) }\end{array}$ \\
\hline Vida media & $6-10$ horas & 45-68 horas & 7 horas \\
\hline
\end{tabular}

Datos tomados de las Fichas Técnicas.

5HMT: 5 hidroximetiltolterodina; a lgp: alfaglicoproteína; Vd: Volumen de distribución;

UPP: unión a proteínas plasmáticas

CYP: Citocromo P-450 * Existen polimorfismos, 7\% de la población son metabolizadores lentos 
rior a las 24 horas que permite una cómoda administración una sola vez al día, explica que el equilibrio estacionario se consiga a los 10 días de tratamiento y hace que el olvido de una dosis no tenga influencia en la respuesta terapéutica (30).

La Tabla III permite la comparación de los principales parámetros farmacocinéticos de los tres antimuscarínicos comercializados más recientemente.

\section{Efecto de la edad y el sexo sobre la farmacocinética.}

Comparados con los jóvenes (20-55 años), las personas de edad avanzada (64-78 años), presentan un incremento de la $A B C$ y la Cmax del orden del $20 \%$ y el $16 \%$ respectivamente. Estos efectos pueden explicarse por un metabolismo más lento y no son clínicamente relevantes $(45,46)$. No hay diferencias por razón de sexo. No es necesario ajustar las dosis en pacientes de edad avanzada y se pueden usar las mismas dosis en hombres que en mujeres.

\section{Efecto de la enfermedad hepática y renal}

En pacientes con insuficiencia hepática moderada (Grado 7-9 de Child-Pugh) la absorción y la Cmax de solifenacina no se afectan, pero se observa un incremento de la biodisponibilidad $(A B C)$ y de la vida media, que son entre 1,6 y 2 veces superiores a las de individuos sanos. Se prolonga también el tiempo para conseguir el equilibrio estacionario. Estos efectos parecen indicar una menor eliminación en estos pacientes, en los que puede ser necesario hacer ajustes de dosis, en todo caso no deberían recibir más de $5 \mathrm{mg}$ una vez al día $(35,44)$. En pacientes con insuficiencia hepática grave no se recomienda el uso de solifenacina $(35,44)$.

En pacientes con insuficiencia renal se produce un incremento de hasta dos veces de la biodisponibilidad $(A B C)$, fundamentalmente por un retraso en la eliminación con prolongación de la $t_{1 / 2}$ (47). Dadas las características cinéticas de solifenacina, en sujetos con insuficiencia renal moderada y aclaramiento de creatinina superior a $30 \mathrm{ml} / \mathrm{min}$ no es necesario hacer ajustes de dosis, en casos de insuficiencia renal grave $(\leq 30 \mathrm{ml} / \mathrm{min}$ de aclaramiento) se debe usar como máximo la dosis de $5 \mathrm{mg} /$ día $(30,35)$.

\section{Interacciones farmacocinéticas}

Han sido estudiados, en hepatocitos humanos aislados, los posibles efectos de solifenacina sobre las isoformas más importantes del citocromo P450 (1 A1 /2, 2C9, 2C19, 2D6 y 3A4) y sobre la P-glicoproteína $(35,48)$. Los resultados indican que solifenacina a concentraciones terapéuticas no tiene efecto sobre estas enzimas, ni sobre el transportador, lo que permite afirmar que solifenacina no modificará la respuesta de otros fármacos que utilicen estas vías metabólicas.

Dado que el citocromo $3 \mathrm{~A} 4$ es muy importante en el metabolismo de solifenacina, se ha investigado si éste se modifica por la administración concomitante de inhibidores potentes como el ketoconazol, o de otros sustratos como warfarina, digoxina o anticonceptivos orales. El tratamiento con ketoconazol durante una semana incrementa en un $50 \%$ el $A B C$ y la Cmax de solifenacina (49). De acuerdo con estos resultados en pacientes tratados con ketoconazol no deberían utilizarse dosis de solifenacina superiores a $5 \mathrm{mg} /$ día, esta precaución debe extenderse a otros inhibidores potentes del CYP 3A4 como otros antifúngicos azólicos, inhibidores de la proteasa o antibióticos macrólidos, en especial eritromicina. No debería utilizarse la asociación en pacientes con insuficiencia hepática moderada o renal grave. Los anticonceptivos orales con etinilestradiol y la warfarina no modifican la farmacocinética de solifenacina $(50,51)$.

El estudio de interacciones con digoxina tiene como objetivo evaluar la influencia de solifenacina sobre la P-glicoproteína, no hay diferencias significativas en los parámetros cinéticos de digoxina y solifenacina después de la administración conjunta, lo que apoya la escasa afinidad del antimuscarínico por el transportador (51).

Solifenacina puede modificar la absorción de otros fármacos como consecuencia de la inhibición de la motilidad intestinal. No obstante, no se han descrito interacciones clínicamente relevantes en este sentido.

El riesgo de interacciones de solifenacina es bajo, e inferior al de los antimuscarínicos que se metabolizan también por el CYP 2D6, en los cuales debe tenerse precaución cuando se administran conjuntamente con inhibidores potentes. Además el riesgo en estos casos es superior para los pacientes metabolizadores lentos.

\section{CONCLUSIÓN}

Los antimuscarínicos son los fármacos de primera elección para el tratamiento del síndrome de vejiga hiperactiva y su relación beneficio/riesgo depende en gran medida de la selectividad por los distintos subtipos de receptores muscarínicos. Solifenacina es el que muestra mayor selectividad por los receptores M3 vesicales, lo que le confiere ventajas sobre otros fármacos del grupo. Solifenacina es metabolizada sólo por el CYP3A4 originado tres 
metabolitos inactivos y uno con actividad similar a la del compuesto original. La fracción inalterada de solifenacina que se elimina por orina junto con el metabolito activo pueden contribuir al efecto terapéutico actuando sobre los receptores del urotelio.

\section{BIBLIOGRAFÍA y LECTURAS RECOMENDADAS (*lectura de interés $y^{* *}$ lectura fundamental)}

1. Abrams P, Cardozo L, Fall M, Griffiths D, Rosier $\mathrm{P}$, Ulmsten U, et al. The standardisation of terminology in lower urinary tract function: report from the Standardisation Sub-committee of the International Continence Society. Urology 2003;61:3749.

2. Milsom I, Abrams P, Cardozo L, Roberts RG, Thueroff J, Wein AJ. How widespread are the symptoms of an overactive bladder and how are they managed? A population-based prevalence study. BJU International 2001;87:760-6.

3. Stewart WF, Van Rooyen JB, Cundiff GW, Abrams P, Herzog AR, Corey R, et al. Prevalence and burden of overactive bladder in the United States. World J Urol 2003;20:327-36.

4. Castro D, Espuña M, Prieto M, Badia X. Prevalencia de Vejiga Hiperactiva en Es-paña: Estudio poblacional. Arch Esp Urol 2005;58:131-8.

*5. Abrams P, Andersson KE, Buccafusco JJ, Chapple C, de Groat WC, Fryer AD, et al. Muscarinic receptors: their distribution and function in body systems, and the implications for treating overactive bladder. Br J Pharmacol 2006;148:565-78

*6. Chapple CR, Yamanishi T, Chess-Williams R. Muscarinic receptor subtypes and management of the overactive bladder Urology 2002;60(Suppl 1):82-8.

7. Chess Williams R. Muscarinic receptors of the urinary bladder: detrusor, urothelial, and projectional. Auton Autocoid Pharmacol 2002;22:133-45.

8. Schneider T, Fetscher C, Krege S, Michel MC. Signal transduction underlying carbachol-induced contraction of human urinary bladder. J Pharmacol Exp Ther 2004;309:1148-53.

**9. Andersson KE, Wein AJ. Pharmacology of the lower urinary tract: basis for current and future treatments of urinary incontinence. Pharmacol Rev 2004;56:581-631.

*10. Stevens LA, Chapple CR, Chess-Williams R. Human idiopathic and neurogenic overactive bladders and the role of $\mathrm{M} 2$ muscarinic receptors in contraction. Eur Urol 2007;52:531-8.

11. Yamaguchi O. Beta3-adrenoceptors in human detrusor muscle. Urology 2002;59(Supp11): 25-9.

*12. Bschleipfer T, Schukowski K, Weidner W, Grando SA, Schwantes U, Kummer W et al. Expres- sion and distribution of cholinergic receptors in the human urothelium. Life Sci 2007;80:2303-7.

13. Yamaguchi $O$. Antimuscarinics and overactive bladder: other mechanism of action. Neurourol Urodyn 2009 Aug 19 [Epub ahead of print PMID: 19693952].

14. Roosen A, Chapple CR, Dmochowski RR, Fowler CJ, Gratzke C, Roehrborn CG, et al. A Refocus on the Bladder as the Originator of Storage Lower Urinary Tract Symptoms: A Systematic Review of the Latest Literature. Eur Urol 2009,56:810-20.

**15. Abrams P, Andersson KE. Muscarinic receptor antagonists for overactive bladder. BJU International 2007;100:987-1006.

16. Westfall DP, Fedan JS, Colby J, Hogaboom GK, O'Donnell JP. Evidence for a contribution by purines to the neurogenic response of the guinea-pig urinary bladder. Eur J Pharmacol 1983;87:41522.

17. de Groat WC. The urothelium in overactive bladder: passive bystander or active participant? Urology 2004;64(Suppl 1):7-11.

18. Andersson KE. LUTS treatment: future treatment options. Neurourol Urodyn 2007;26:934-47.

19. Andersson KE, Fullhase C, Soler R. Urothelial effects of oral agents for overactive bladder. Curr Urol Rep 2008;9:459-64.

20. Andersson KE, Yoshida M. Antimuscarinics and the overactive detrusor - which is the main mechanism of action? Eur Urol 2003;43:1-5.

21. Reynard JM. Does anticholinergic medication have a role for men with lower urinary tract symptoms / benig prostatic hyperplasia either alone or in combination with other agents? Curr Opin Urol 2004;14:13-6.

*22. Hedge SS. Muscarinic receptors in the bladder: from basic research to therapeutics. Br J Pharmacol 2006;147:S80-7.

23. Hoffstetter S. Solifenacin succinate for the treatment of overactive bladder. Expert Opinion on Drug Metabolism \& Toxicology 2009;5:345-50.

**24. Ohtake A, Saitoh C, Yuyama H, Ukai M, Okutsu $\mathrm{H}$, Noguchi Y, et al. Pharmacological characterization of a new antimuscarinic agent, solifenacin succinate, in comparison with other antimuscarinic agents. Biol Pharm Bull 2007;3054-8.

25. Michel MC. Fesoterodine: a novel muscarinic receptor antagonist for the treatment of overactive bladder syndrome. Expert Opin Pharmacother 2008;9:1787-96.

26. Okutsu H, Noguchi Y, Ohtake A, Suzuki M, Sato $S$, Sasamata $M$ et al. Effects of intravenously and orally administered solifenacin succinate (YM905) on carbachol-induced intravesical pressure elevation and salivary secretion in mice. Biol Pharm Bull 2007;30:2324-7

*27. Ikeda K, Kobayashi S, Suzuki M, Miyata K, 
Takeuchi M, Yamada T, et al. M(3) receptor antagonism by the novel antimuscarinic agent solifenacin in the urinary bladder and salivary gland. Naunyn Schmiedebergs Arch Pharmacol 2002;366:97-103.

28. Ohtake A, Ukai M, Hatanaka T, Kobayashi S, Ikeda $\mathrm{K}$, Sato $\mathrm{S}$, et al. In vitro and in vivo tissue selectivity profile of solifenacin succinate YM905 for urinary bladder over salivary gland in rats. Eur J Pharmacol 2004;492:243-50.

29. Kobayashi S, Ikeda K, Miyata K. Comparison of in vitro selectivity profiles of solifenacin succinate YM905 and current antimuscarinic drugs in bladder and salivary glands: a Ca2+ mobilization study in monkey cells. Life Sci 2004;74:843-53.

30. Doroshyenko O, Fuhr U. Clinical Pharmacokinetics and Pharmacodynamics of Solifenacin. Clin Pharmacokinet 2009;48:281-302.

31. Chuang YC, Thomas CA, Tyagi S, Yoshimura N, Tyagi P, Chancellor MB. Human urine with solifenacin intake but not tolterodine or darifenacin intake blocks detrusor overactivity Int Urogynecol J Pelvic Floor Dysfunct 2008;19:1353-7.

*32. Smulders RA, Krauwinkel WJ, Swart PJ, Huang M. Pharmacokinetics and safety of solifenacin succinate in healthy young men. J Clin Pharmacol 2004;44:1023-33.

33. Kuipers ME, Krauwinkel WJ, Mulder H, Visser N. Solifenacin demonstrates high absolute bioavailability in healthy men. Drugs R D 2004;5:73-81.

34. Krauwinkel WJ, Smulders RA, Mulder H, Swart PJ, Taekema-Roelvink ME. Effect of age on the pharmacokinetics of solifenacin in men and women. Int J Clin Pharmacol Ther 2005; 43:227-38.

35. Vesicare (Solifenacina) Ficha Técnica.

https://sinaem4.agemed.es/consaem/especialidad. do? metodo $=$ verFichaWordPdf $\&$ codigo $=66258 \&$ formato $=$ pdf $\&$ formulario $=$ FICHAS $[$ Consultado 16 octubre 2009].

36. Uchida T, Krauwinkel WJ, Mulder H, Smulders RA. Food does not affect the pharmacokinetics of solifenacin, a new muscarinic receptor antagonist: results of a randomized crossover trial. Br J Clin Pharmacol 2004;58:4-7.

37. Brynne N, Stahl MMS, Hallen B, Edlund PO, Palmér L, Höglund P, et al. Pharmacokinetics and pharmacodynamics of tolterodine in man: a new drug for the treatment of urinary bladder overactivity. Int J Clin Pharmacol Ther 1997;35:287-95.

38. Douchamps J, Derenne F, Stockis A, Gangji D, Juvent M, Herchuelz A. The pharmacokinetics of oxybutynin in man. Eur J Clin Pharmacol 1988; 35:515-20.

*39. Maniscalco M, Singh-Franco D, Wolowich WR, Torres-Colón R. Solifenacin succinate for the treatment of symptoms of overactive bladder. Clin Ther 2006;28:1247-72.
40. Wesnes KA, Edgar C, Tretter RN, Bolodeoku J. Exploratory pilot study assessing the risk of cognitive impairment or sedation in the elderly following single doses of solifenacin $10 \mathrm{mg}$. Expert Opin Drug Saf 2009 Sep 11. [Epub ahead of print]

*41. Kay GG, Abou-Donia MB, Messer WS Jr, Murphy DG, Tsao JW, Ouslander JG. Antimuscarinic drugs for overactive bladder and their potential effects on cognitive function in older patients. $\mathrm{J}$ Am Geriatr Soc 2005;53:2195-201.

42. Detrusitol Neo (Tolterodina). Ficha técnica https://sinaem4.agemed.es/consaem/especialidad. do? metodo $=$ verFichaWordPdf \& $\operatorname{codigo}=64245 \&$ formato $=$ pdf $\&$ formulario $=$ FICHAS [Consultado 16 octubre 2009].

43. Toviaz (Fesoterodina). Ficha técnica. http://www.emea.europa.eu/humandocs/PDFs/ EPAR/toviaz/emea-combined-h723es.pdf [Consultado 16 octubre 2009].

44. Kuipers M, Smulders R, Krauwinkel W, Hoon T. Open-label study of the safety and pharmacokinetics of solifenacin in subjects with hepatic impairment. J Pharmacol Sci 2006;102: 405-12.

45. Dawling S, Crome P. Clinical pharmacokinetic considerations in the elderly: an update. Clin Pharmacokinet 1989;17:236-63.

46. Hammerlein A, Derendorf H, Lowenthal DT. Pharmacokinetic and pharmacodynamic changes in the elderly: clinical implications. Clin Pharmacokinet 1998;35:49-64.

47. Smulders RA, Smith NN, Krauwinkel WJ, Hoon, T. Pharmacokinetics, safety, and tolerability of solifenacin in patients with renal insufficiency. J Pharmacol Sci 2007;103:67-74.

48. Michel MC, Yanagihara T, Minematsu T, Swart PJ, Smulders RA. Disposition and metabolism of solifenacin in humans. Br J Clin Pharmacol 2005;59:647.

49. Swart PJ, Krauwinkel WJ, Smulders RA, Smith NN. Pharmacokinetic effect of ketoconazole on solifenacin in healthy volunteers. Basic Clin Pharmacol Toxicol 2006;99:33-6.

50. Taekema-Roelvink ME, Swart PJ, Kuipers ME, Krauwinkel WJ, Visser N, Smulders RA.Pharmacokinetic interaction of solifenacin with an oral contraceptive containing ethinyl estradiol and levonorgestrel in healthy women: a double-blind, placebo-controlled study. Clin Ther 2005;27:1403-10.

51. Smulders RA, Kuipers ME, Krauwinkel WJ. Multiple doses of the antimuscarinic agent solifenacin do not affect the pharmacodynamics or pharmacokinetics of warfarin or the steady-state pharmacokinetics of digoxin in healthy subjects. Br J Clin Pharmacol 2006;62:210-7. 